\title{
Analytical Successive Solutional Fractionation of Macromolecules
}

\author{
Yukio Miyazaki and Kenji Kamide* \\ Textile Research Laboratory, Asahi Chemical Industry Co. Ltd., \\ Takatsuki, Osaka, Japan.
}

(Received August 30, 1976)

\begin{abstract}
A detailed theoretical comparison between the efficiencies of the analytical successive solutional fractionation (SSF) and analytical successive precipitational fractionation (SPF) was made under various conditions of the average molecular weight of original polymers, the molecular weight distribution (MWD), the initial polymer volume fraction, and the total number of fractions by using a computer simulation technique. The results were compared with the experimental results obtained for an atactic polystyrene solution in methylcyclohexane. The limit of applicability of the Schulz method and Kamide methods as analytical procedures was obtained.

SSF proved successful for evaluating the total profile of MWD of the original polymer. In particular, the combination of SSF with the Kamide method furnished the most accurate procedure for evaluating MWD curve of the original polymer under ordinary conditions. The operating conditions suitable for determining $\bar{X}_{w} / \bar{X}_{n}$ of the original polymer were, however, extremely limited as compared with those in the preparative fractionation.
\end{abstract}

KEY WORDS Analytical Successive Solutional Fractionation / Average Molecular Weight / Molecular Weight Distribution / Computer Simulating Technique /

The molecular weight fractionation by solubility difference has been extensively employed for many years as a principal method for evaluating the molecular weight distribution (MWD) of macromolecules. Due to rapid development of gel permeation chromatography (GPC), the frequency of utilization of the fractionation method has decreased recently as a mean of in-plant process control. An exact evaluation of MWD by GPC is possible only in two cases when (1) nearly monodisperse and well-characterized polymer samples are available as standard materials for calibrating the GPC curve obtained and (2) the hydrodynamic volume universal calibration plot is strictly justified for the polymer type of interest. Unfortunately, very few polymers except atactic polystyrene are commercially supplied as standard materials and even if standard samples are available, optimiza-

* To whom the correspondence should be addressed. tion of the experimental conditions as well as rigorous band broadening correction is neccessary. ${ }^{1}$ Recent studies ${ }^{2-6}$ on the application limit of the universal plot revealed that the plot is not universal when a second separation mechanism exists in addition to steric exclusion separation and when all the polymer samples have different molecular geometrics and rigidities. It seems sufficient to note that the efficiency of GPC fractionation can not be thoroughly studied without the aid of a complete theory, which has not yet been established. In short, GPC is not almighty, but has the following drawbacks to be improved in the future; (1) expensive apparatus, (2) high running, cost (3) narrow choice of solvents and as a result danger to the operator's health from solvent toxicity (4) severe limitation of polymer types for which MWD can be evaluated by GPC.

In contrast to GPC, fractionation by the solubility difference now has several merits such as (1) cheap equipment, (2) wide choice of sol- 
vent, (3) relatively small amount of solvent needed to obtain for the sample (as compared with GPC and column fractionation) and (4) definite theoretical background. Some people hold the opinion that analytical use of fractionation by solubility difference is now out of data and meaningless. However, the importance of analytical successive fractionation by solubility difference never diminishes as is evident from the above discussion and even in the future this classical method has, we believe, a good chance of continued use in a research laboratory for analytical purposes.

Molecular weight fractionation by solubility difference (without exception, successive precipitational fractionation (SPF)), has been widely used as a method for studying polymerization mechanism and consequently, a large amount of fractionation data has been accumulated. ${ }^{7}$ We regret to say that no careful attention was paid to the analytical procedure not to the accuracy of the results obtained. If the reliability of MWD curves constructed from SPF data is made clear, these literature data are expected to play a much more important role in determin- ing polymerization mechanisms. In this sense, it is very important to throw light on the reliability of analytical fractionation method by solubility difference in relation to the operating conditions of the fractionation and to the analytical procedure adopted.

The extent of uncertainty inherent in this method can be clarified in the following sophisticated manner; A computer simulated fractionation is carried out, on the basis of theory, which is rigorously valid, on a given polymer of predetermined MWD under defined operating conditions (fractionation procedure, initial polymer volume fraction $v_{\mathrm{p}}{ }^{0}$, the fraction size $\rho$, or the total number of fractions $n_{\mathrm{t}}$ ). MWD of the original polymer is evaluated from the fractionation data and is compared with the true MWD which is known in advance.

The theoretical studies following the line of the above discussion were performed by Matsumoto and Ohyanagi (MO), ${ }^{8}$ Booth and Beason (BB), ${ }^{9}$ Tung, ${ }^{10}$ and Kamide, et al. ${ }^{11}$ The details are summerized in Table I. Among them, Kamide and his coworkers established the modern theory of phase separation and of fractionation

Table I. Theoretical studies of accuracy of the fractional method by the solubility difference

\begin{tabular}{|c|c|c|c|c|c|}
\hline & \multicolumn{5}{|c|}{ Reporter } \\
\hline & $\begin{array}{l}\text { Matsumoto-- } \\
\text { Ohyanagi }\end{array}$ & $\begin{array}{l}\text { Booth- } \\
\text { Beason }\end{array}$ & Tung & Kamide, & et al. \\
\hline Theory & $\mathrm{FH}^{\mathrm{a}}$ & FH & FH & Modified & $\mathrm{FH}^{\mathrm{b}}$ \\
\hline Procedure & $\mathrm{SPF}^{\mathrm{c}}$ and $\mathrm{SSF}^{\mathrm{d}}$ & SPF & SPF & SPF & SSF \\
\hline Original polymer & $\bar{X}_{n}=500^{\circ}$ & $\bar{X}_{n}=5$ & $\bar{X}_{w}=50^{\mathrm{f}}$ & $\begin{array}{l}\bar{X}_{w}=300-3000 \\
\bar{X}_{w} / \bar{X}_{n}=1.05-5 \\
\mathrm{SZ}^{\mathrm{g}} \text { and } \mathrm{W}^{\mathrm{h}}\end{array}$ & $\begin{array}{l}\bar{X}_{w}=300 \\
\bar{X}_{w /} / \bar{X}_{n}=2 \\
\mathbf{S Z}\end{array}$ \\
\hline Operating conditions & $\begin{array}{l}R=10^{2 \mathrm{i}} \\
n_{\mathrm{t}}=8^{\mathrm{j}}\end{array}$ & $\begin{array}{l}R=10^{2} \\
n_{\mathrm{t}}=5\end{array}$ & $\begin{array}{l}v_{\mathrm{p}}^{0}=0.5 \text { and } 2 \% \mathrm{k} \\
n_{\mathrm{t}}=10\end{array}$ & $\begin{array}{l}v_{\mathrm{p}}^{0}=0.01-1 \% \\
n_{\mathrm{t}}=4-20 \\
p=0 \text { and } 0.5^{1}\end{array}$ & $\begin{array}{l}v_{\mathrm{p}}{ }^{0}=1 \text { and } 5 \\
n_{\mathrm{t}}=15 \\
p=0 \text { and } 0.5\end{array}$ \\
\hline $\begin{array}{l}\text { Numbers of } \\
\text { fractionation runs }\end{array}$ & 2 & 1 & 2 & 17 & 3 \\
\hline Analytical method & $\mathbf{S}^{\mathrm{m}}$ & $\mathbf{S}$ & $\mathbf{S}$ & $\mathbf{S}$ and $\mathrm{K}^{\mathrm{n}}$ & $\mathrm{S}$ and $\mathrm{K}$ \\
\hline Evaluation & $\mathrm{q}^{\circ}$ & $\mathrm{q}$ & $\mathrm{q}$ & $E^{\mathrm{p}}$ and $E^{\prime \mathrm{q}}$ & \\
\hline
\end{tabular}

a Flory-Huggins, b concentration dependence of polymer-solvent interaction parameter expressed by $\mathrm{p}$ was taken into account for the original FH theory, c successive precipitational fractionation, d successive solutional fractionation, e number-average degree of polymerization, $f$ weight-average degree of polymerization, g Schulz-Zimm distribution, $\mathrm{h}$ Wesslau distribution, i volume ratio of polymer-lear phase to polymer-rich phase, $j$ total number of fractions in a run, $k$ initial polymer volume fraction, ${ }^{1} p$ is defined in eq 1 , $\mathrm{m}$ Schulz, ${ }^{n}$ Kamide, ${ }^{\circ}$ qualitative, ${ }^{p} E$ is defined by eq 6 , q $E^{\prime}$ is defined by eq 13 . 
by solubility difference, where the concentration dependence of the polymer-solvent thermodynamics interaction parameter $\chi$ of the original Flory-Huggins $(\mathrm{FH})$ theory was properly taken into account;

$$
\chi=\chi_{0}\left(1+p v_{\mathrm{p}}\right)
$$

where $\chi_{0}$ and $p$ are constants related to solvent nature and $v_{\mathrm{p}}$ is the polymer volume fraction. ${ }^{11-17}$ Kamide, et al's theory received ample experimental support. ${ }^{18-20}$ In particular, in our previous paper ${ }^{20}$ we performed, on the basis of the modern theory, a hypothetical fractionation using an electronic computer on a polymer having the same MWD as that used for the practical experiment under identical operating conditions with experiment and calculated the partition coefficient $\sigma$, the volume ratio $R$, the polymer volume fractions in both phases, and MWD as well as $\bar{M}_{w} / \bar{M}_{n}$ of the polymer partitioning in two immiscible phases. The excellent agreement between the experimental and theoretical values of these various parameters was confirmed conclusively.

In this manner, we concluded that the fractionation theory and the simulative technique must be accepted with any reserve to be accurate and quantitative in nature. On this basis, the analytical successive precipitational fractionation (SPF) has been studied in a very systematic manner and definite conclusions have been reached. In contrast, although analytical successive solutional fractionation (SSF) is expected to be much superior to analytical SPF $^{12-15,17,21}$ the theoretical studies of analytical SSF are qualitative and not comprehensive. Therefore, the conclusions covering more general cases are required.

This paper intends to clarify the effect of the molecular characteristics of the original polymer and of the operating condition, including initial polymer volume fraction and the total number of fractions, on the accuracy of analytical SSF in comparison with analytical SPF and to verify the validity of the approximation adopted in Kamide's method, by actual experiment on the polystyrene/methylcyclohexane system.

\section{Molecular Characteristics of the Original Polymer and Simulative Procedure}

The modified FH solution theory was em- ployed as a basic principle, where the concentration dependence of $\chi$ was taken into consideration. The polymers with weight-average degree of polymerization $\bar{X}_{w}{ }^{0}$ (hereafter referred to as $\bar{X}_{w}{ }^{0}$ ) of 150 to 3000 and the ratio of the weight- to number-a verage degree of polymerization $\bar{X}_{w} / \bar{X}_{n}$ of 1.05 to 5 , having both SchulzZimm and Wesslau distributions, were dissolved in a suitable single solvent to provide solutions with volume fractions of polymer $v_{\mathrm{p}}{ }^{0}$ from 0.01 to $10 \%$. Quasi-binary mixtures thus prepared were then subjected to hypothetical SSF or SPF (if necessary), according to the method of Kamide and Sugamiya. ${ }^{22}$

The standared conditions adopted throughout this paper are: Schulz-Zimm distribution, $\bar{X}_{w}{ }^{0}=300$, SZ 2 (The Schulz-Zimm distribution with $\bar{X}_{w} / \bar{X}_{n}=2$ is hereafter abbreviated to SZ 2), $v_{\mathrm{p}}{ }^{0}=1 \%$ and the total number of fractions in a given run $n_{\mathrm{t}}=15$. If the effect of one of the factors mentioned-above on the relative error $E$ (see eq 6) is to be examined, the standard conditions are chosen for all the other factors unless otherwise stated. In a series of theoretical studies, ${ }^{11-15}$ it has been ascertained that the conclutions deduced under the standard conditions have a general character.

\section{Method for Evaluating the MWD of the Original Polymer \\ Using the fractionation data $\left(\rho\right.$ and $\left.\bar{X}_{w}\right)$ the} MWD of the original polymer can be evaluated by means of the widely-accepted method of Schulz $^{23}$ and by means of a method proposed on the basis of the modern fractionation theory by Kamide and his coworkers. ${ }^{21,24}$ These can be summarized in short as follows:

(1) Method of Schulz. The cumulative weight fraction, $I\left(\bar{X}_{w, j}\right)$, corresponding to $\bar{X}_{w, j}$ of the $j$ th fraction is given by

$$
I\left(\bar{X}_{w, j}\right)=\sum_{k=j+1}^{n_{\mathrm{t}}} \sum_{X=0}^{\infty} g_{k}(X)+1 / 2 \sum_{X=0}^{\infty} g_{j}(X)
$$

for SPF (2)

or

$$
\begin{array}{r}
I\left(\bar{X}_{w, j}\right)=\sum_{k=1}^{j-1} \sum_{X=0}^{\infty} g_{k}(X)+1 / 2 \sum_{X=0}^{\infty} g_{j}(X) \\
\text { for SSF }
\end{array}
$$

where $\sum_{X=0}^{\infty} g_{k}(X)$ denotes the weight fraction of the $k$ th fraction. 
(2) Method of Kamide, et al., for SPF and $S S F$. MWD of the fraction separated by SPF can be represented with fairly good accuracy by the equilateral triangle having its peak at $\bar{X}_{w}$ in the range, $0 \leq \bar{X}_{w} \leq 2 \bar{X}_{w}$ (Figure 1a). The distribution function of the $j$ th fraction, $g_{j}(X)$ can be expressed as

$$
g_{j}(X)=\frac{\left(\sum_{X=0}^{\infty} g_{j}(X)\right) X}{\bar{X}_{w, j}^{2}} \text { for } 0 \leq X \leq \bar{X}_{w, j}
$$

and

$$
\begin{aligned}
g_{j}(X)=\frac{\left(\sum_{X=0}^{\infty} g_{j}(X)\right)\left(2 \bar{X}_{w, j}-X\right)}{\bar{X}_{w, j}^{2}} \quad \text { for } \bar{X}_{w, j} \leq X<2 \bar{X}_{w, j}
\end{aligned}
$$

and

$$
g_{j}(X)=0 \text { for } 2 \bar{X}_{w, j} \leq X
$$

here, $\left(\sum_{X=0}^{\infty} g_{j}(X)\right)$ denotes the weight fraction of the $j$ th fraction to the total weight of polymer. The summation of $g_{j}(X)\left(=\sum_{j=1}^{n} g_{j}(X)\right)$ given by eq 3-5 with respect to $X$ provides the normalized differential MWD, $g_{\mathrm{e}}(X)$, of the original polymer estimated from the fractionation data and its integration yields the cumulative distribution curve, $I_{\mathrm{e}}(X)$.

An improvement of the original method of Kamide, et al., for the case of SSF can be made by introducing the modified triangle distribution, ranging from $(1 / 2) X_{\mathrm{p}}$ to $2 X_{\mathrm{p}}$, where $X_{\mathrm{p}}$ is the

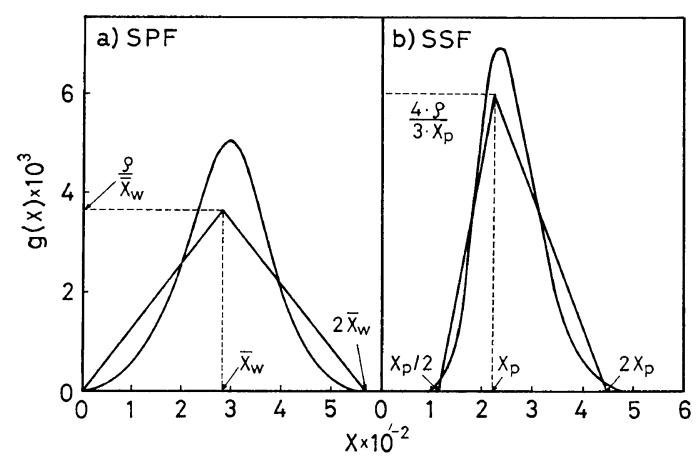

Figure 1. Schematic representation of triangleshaped MWD (see text). Full lines are the true MWD of the fraction: a) successive precipitational fractionation (SPF); b) successive solutional fractionation (SSF). value of $X$ at peak and the triangle has the value of $\bar{X}_{w}$ identical with that of the fraction separated (Figure 1b).

A parameter $E$ defined by eq 6 is utilized as a measure of the accuracy of the methods for estimating the MWD of the original polymer:

$$
E=\int\left|g_{0}(X)-g_{\mathrm{e}}(X)\right| \mathrm{d} x
$$

here, $g_{0}(X)$ is the normalized true differential MWD of the original polymer. $\left|g_{0}(X)-g_{\mathrm{e}}(X)\right|$ is the absolute magnitude of the difference between $g_{0}(X)$ and $g_{\mathrm{e}}(X)$.

For convenience, the combination of the fractionation procedure (SPF or SSF) and the analytical method (Schulz or Kamide) will be referred to hereafter; for example, SPF/Schulz (or SSF/Kamide) means that the fractionation data obtained by SPF (or SSF) are treated by the Schulz (or Kamide) methods.

\section{Fractionation Experiment}

A sample of atactic polystyrene was fractionated from a $0.94-\%$ (vol $\%$ ) solution in methylcyclohexane into 14 and 23 fractions by SSF and into 13 and 18 fractions by SPF. The fractions isolated thus, as well as those obtained in the preceding paper, ${ }^{20}$ were analyzed by GPC. The detailed measuring conditions and the method of correcting column band spreading have already been described elsewhere. ${ }^{18}$ At the same time computer simulation of SSF and SPF was carried out for this polymer under the same operating conditions as those in the actual experiment. In this case, the parameter $p$, was taken as $0.7 .^{20}$

\section{RESULTS AND DISCUSSION}

Effect of Average Molecular Weight and Polydispersity of the Original Polymer

A plot of the relative error $E v s . \bar{X}_{w}{ }^{0}$ is shown in Figure 2, where the standard conditions described before, except for $\bar{X}_{w}{ }^{0}$, were utilized. From inspection of Figure 2 the accuracy of an analytical fractionation appears to be much more dependent on the analytical method than the operating conditions. The average molecular size of the original polymer has a negligible effect on peculiar features of both methods for estimating the MWD in the original polymer 


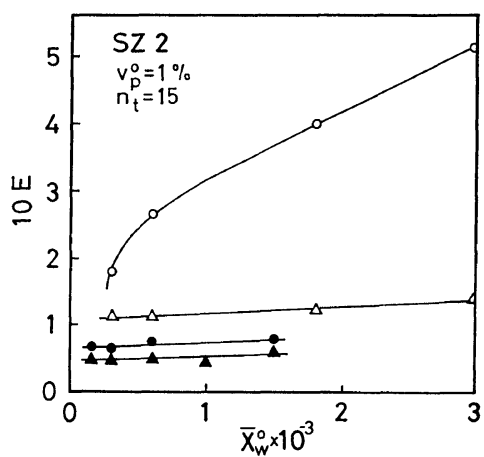

Figure 2. Effect of the weight-average degree of polymerization of the original polymer $\bar{X}_{w}{ }^{0}$ on the relative error $E$. In this case $g_{\mathrm{e}}(X)$ in eq 6 was estimated from the fractionation data by SPF open mark) or SSF (closed mark) by use of the Kamide method (triangel) or the Schulz method (circle). The original polymer, Schulz-Zimm distribution, SZ 2, $p=0, n_{\mathrm{t}}=15, v_{\mathrm{p}}{ }^{0}=1 \%$.

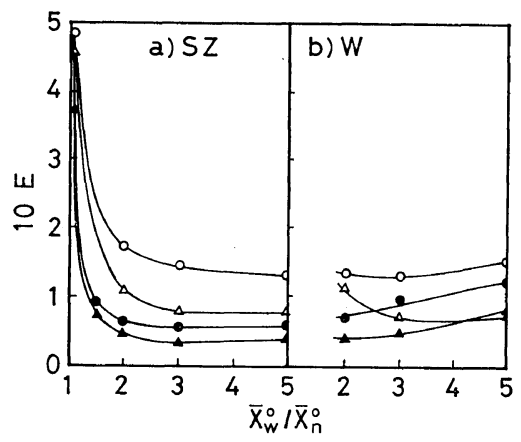

Figure 3. Effect of the polydispersity of the original polymer, $\bar{X}_{w}{ }^{0} / \bar{X}_{n}{ }^{0}$ on the relative error $E$. The marks in figures have the same meaning as those in Figure $2\left(v_{\mathrm{p}}^{0}=1 \%, n_{\mathrm{t}}=15, p=0\right)$ : a) the Schulz-Zimm type polymer $\left(\bar{X}_{w^{0}}=300\right)$; b) the Wesslau type polymer $\left(\bar{X}_{w}{ }^{0}=300\right)$.

from the fractionation data, except for the case of SPF/Schulz method. ${ }^{23}$ There is a marked difference in $E$ between SSF and SPF, suggesting the superiority of SSF over SPF.

In Figure 3 the relative error $E$ is plotted as a function of $\bar{X}_{w}{ }^{0} / \bar{X}_{n}{ }^{0}$ of the original polymer where the polymer having the Schulz-Zimm or Wesslau distributions $\bar{X}_{w}{ }^{0}=300$ was fractionated into fifteen equal fractions from a 1.0-\% solution. With progressively increasing $\bar{X}_{w}{ }^{0} / \bar{X}_{n}{ }^{0}$ the accuracy of the methods for estimating the original MWD improves considerably irrespec- tive of the type of MWD (Schulz-Zimm distribution or Wesslau distribution). This appears to be mainly a consequence of the fact that the approximations adopted in both treatments become more accurate as $\bar{X}_{w}{ }^{0} / \bar{X}_{n}{ }^{0}$ increases. At least as far as a 1.0-\% polymer solution is concerned, the Kamide method is preferable to the Schulz method, regardless of the type and breadth of the MWD in the original polymer. For a very narrow distribution polymer (e.g., a polymer having $\bar{X}_{w}{ }^{0} / \bar{X}_{n}{ }^{0}$ smaller than 1.10) $g_{\mathrm{e}}(X)$ or $I_{\mathrm{e}}(X)$ evaluated by using the above methods deviated very significantly from the true original MWD. This has been observed, qualitatively, in the case of SPF. ${ }^{11}$

\section{Effect of Initial Polymer Volume Fraction}

The dependence of the accuracy of the two evaluating methods on $v_{\mathrm{p}}{ }^{0}$ is shown in Figure 4. In both SSF and SPF, $E$ obtained by the Kamide method exhibits an intense (in SPF) or a poorly defined (in SSF) minimum at a specific value of $v_{\mathrm{p}}{ }^{0}$. On the other hand $E$ by the Schulz method decreases as $v_{p}{ }^{0}$ decreases. For example, for the polymer with Schulz-Zimm distribution $\left(\bar{X}_{w}{ }^{0}=300\right.$ and $\left.\bar{X}_{w}{ }^{0} / \bar{X}_{n}{ }^{0}=2\right)$ the Kamide method is more accurate at $v_{\mathrm{p}}^{0} \geq 1 \%$, but the Schulz method becomes adequate at $v_{\mathrm{p}}{ }^{0} \leq 0.1 \%$. However, for the Wesslau type polymer the Kamide method is more accurate than the Schulz method over a whole range of $v_{p}{ }^{0}$. The superiority of

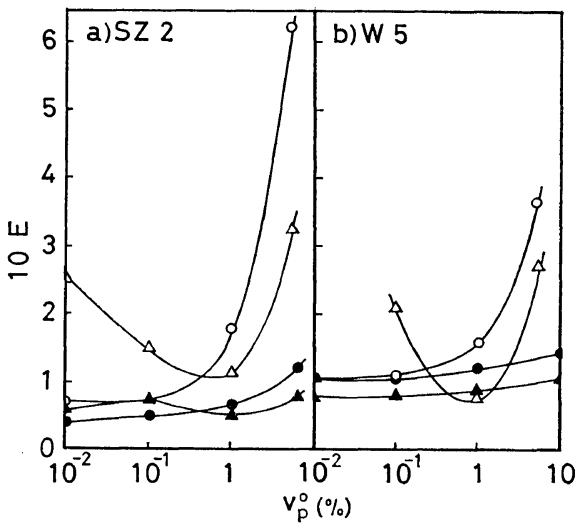

Figure 4. Effect of the initial polymer volume fraction $v_{\mathrm{p}}{ }^{0}$ on the relative error $E$. The marks in the figures have the same meaning as those in Figure $2\left(n_{\mathrm{t}}=15, p=0\right)$ : a) the Schulz-Zimm type polymer $\left.\left(\bar{X}_{w}{ }^{0}=300, \mathrm{SZ} 2\right) ; \mathrm{b}\right)$ the Wesslau type polymer $\left(\bar{X}_{w}{ }^{0}=300, \mathrm{~W} 5\right)$. 


\section{Y. Miyazaki and K. Kamide}

SSF over SPF is maintained over a whole range of $v_{\mathrm{p}}^{0}$ from $10^{-2}$ to $10 \%$, irrespective of the analytical procedures employed.

\section{Effect of Total Number of Fractions}

The effect of the total number of fractions $n_{\mathrm{t}}$ on the relative error $E$ is demonstrated in Figure 5. In the both methods, $E$ decreases sharply at first and then approaches a limiting values as $n_{\mathrm{t}}$ increases progressively. It is immediately evident from Figure 5 that the Kamide method is preferable to the Schulz method, at a given $n_{\mathrm{t}}$ at least in the range $n_{\mathrm{t}}>10$, as far as the polymer with the Wesslau distribution $\left(\bar{X}_{w}{ }^{0}=300\right.$ and W 5 (The symbol W 5 represents the Wesslau distribution with $\bar{X}_{w}{ }^{0} / \bar{X}_{n}{ }^{0}=5$.)) is concerned. In the previous paper ${ }^{25}$ it was concluded from inspection of the cumulative weight distribution $I_{\theta}(X)$ of the original polymer, constructed from the data by SPF with SZ2, that in contrast to the Schulz plot, the Kamide plot approaches the true distribution of the original polymer with increasing $n_{\mathrm{t}}$. The quantitative analysis of the fractionation data, obtained by SSF with SZ 2, as shown in Figure 5 indicates that there is no difference, in the effect of $n_{t}$, between the Schulz method and the Kamide method.

\section{Effect of $p$}

The effect of $p$ on $E$ is demonstrated in Figure 6. With an increase in $p, E$ decreases in SPF/ Schulz, but $E$ increases in SPF/Kamide. The

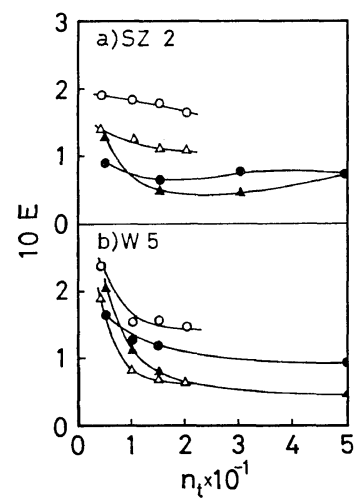

Figure 5. Effect of the total number of fractions in a given run, $n_{t}$, on the relative error $E$. The marks in figure have the same meaning as those in Figure 2: a) the Schulz-Zimm type polymer $\left(\bar{X}_{w^{0}}=300\right.$, SZ 2$) ;$ b) the Wesslau type polymer $\left(\bar{X}_{w^{0}}=300\right.$, W 5$)$.

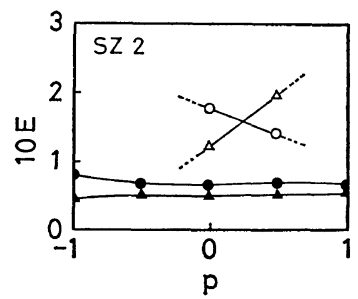

Figure 6. Effect of the concentration dependence of the polymer/solvent interaction parameter $\chi$, $p$, on the relative error $E$ (the original polymer, Schulz-Zimm distribution; $\bar{X}_{w}=300, \mathrm{SZ} 2, v_{\mathrm{p}}{ }^{0}=$ $\left.1 \%, n_{\mathrm{t}}=15\right)$. The marks in the figure have the same meaning as those in Figure 2.

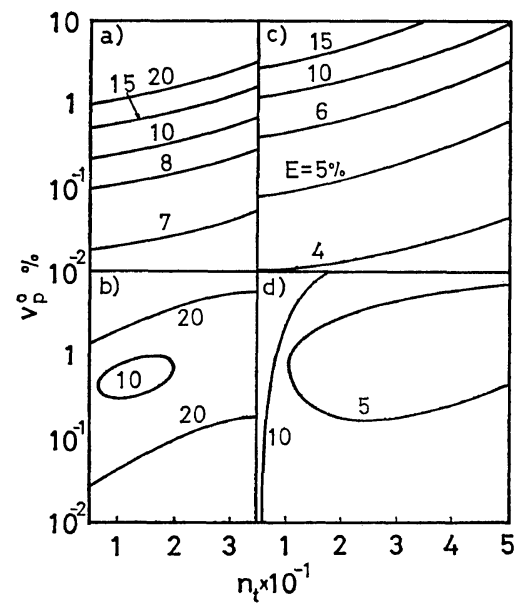

Figure 7. Correlation between the relative error $E$, initial polymer volume fraction $v_{\mathrm{p}}{ }^{0}$, and total number of fractions $n_{\mathrm{t}}$ (the original polymer, Schulz-Zimm distribution, $\bar{X}_{w}{ }^{0}=300$, SZ 2, $p=0$ ): a) SPF/Schulz; b) SPF/Kamide; c) SSF/Schulz; d) SSF/Kamide.

effect of $p$ (i.e., the effect of the type of solvent employed in the fractionation run) is almost significant in the case of SSF. Over a wide range of $p, \mathrm{SSF} / \mathrm{Kamide}$ is the most accurate.

The Optimum Operating Conditions for Analytical $S P F$ and $S S F$

Figure 7 shows the relationships between $v_{\mathrm{p}}{ }^{0}$, $n_{\mathrm{t}}$, and $E$ for a polymer with Schulz-Zimm distribution $\left(\bar{X}_{w}{ }^{0}=300\right.$ and SZ2). The curves in Figure 7 denote countour lines, relating $v_{\mathrm{p}}{ }^{0}$ to $n_{\mathrm{t}}$, for a fixed value of $E$.

The relative error $E$ obtained by the Schulz method approaches close to zero if $v_{\mathrm{p}}{ }^{0} \rightarrow 0$ and 
$n_{t} \rightarrow \infty$ concurrently realized. In contrast to this, $E$ exhibits a remarkable minimum $E_{\mathrm{min}}$ if the Kamide method is employed (in this case, $E_{\min }=$ $10 \%$ for SPF/Kamide and $E_{\min }=4.8 \%$ for SSF/ Kamide, respectively). By use of a Figure like Figure 7 we can optimise the fractionation procedure for analytical purposes for any polymer sample.

Effect of Average Molecular Weight of the Fractions

The average molecular weights commonly utilized in the analytical fractionation are $\bar{X}_{n}$ and the viscosity-average degree of polymerization $\bar{X}_{v}$ besides $\bar{X}_{w}$. If we define $X(\boldsymbol{r})$ by

$$
X(r)=\left[\frac{\int X^{r} g_{0}(X) \mathrm{d} X}{\int g_{0}(X) \mathrm{d} X}\right]^{1 / r}
$$

we can readily express $\bar{X}_{n}, \bar{X}_{v}$, and $\bar{X}_{w}$ in terms of $X(r)$ :

$$
\bar{X}_{n}=X(-1), \quad \bar{X}_{v}=X(a) \quad \text { and } \quad \bar{X}_{w}=X(1)
$$

where $a$ is the exponent in the Mark-HouwinkSakurada equation. It should be noted that $r=0$ is a singular point in eq 8 .

Figure 8 shows the effect of $r$ in $X(r)$ on $E$ for analytical SPF and SSF, where the original polymer with Schulz-Zimm distribntion $\left(\bar{X}_{w}{ }^{0}=\right.$ 300 and $\left.\bar{X}_{w}{ }^{0} / \bar{X}_{n}{ }^{0}=2\right)$ was fractionated from a 1.0-\% solution into 15 equal fractions. The effect of the nature of the average (in this case, $r$ in eq 7) on the molecular weight of fractions

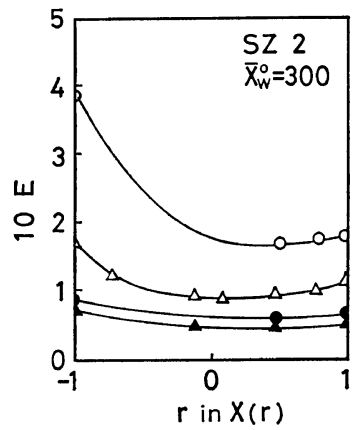

Figure 8. Effect of the moment $r$ in $X(r)$ in eq 8 on the relative error $E$ (the original polymer, Schulz-Zimm distribution, $\bar{X}_{w}{ }^{0}=300$, SZ 2, $v_{\mathrm{p}}{ }^{0}=$ $\left.1 \%, n_{\mathrm{t}}=15\right)$. The marks in the figure have the same meaning as those in Figure 2. becomes pronounced in SPF. For example in the case of SPF/Schulz the value of $E$ doubles if $\bar{X}_{n}$ is employed in place of $\bar{X}_{w}$. The charge in $E$ of SPF if $\bar{X}_{v}$ is used instead of $\bar{X}_{w}$ is, however, negligible. Contrary to this, the dependence of $E$ is SSF on $r$ in $X(r)$ is small. It is very interesting to notice that the usage of $X(r) \quad(0<r \leq 1)$ gives the minimum relative error irrespective of the fractionation method and the analytical procedure.

Correlationships between the Estimated Value of $\bar{X}_{w} / \bar{X}_{n}$ and $E$

The correlation between the value of $\bar{X}_{w} / \bar{X}_{n}$, estimated from the fractionation data and the relative error $E$ is shown in Figure 9, where the polymer with the Schulz-Zimm distribution $\left(\bar{X}_{w}{ }^{0}=300\right.$ and $\left.\bar{X}_{w}{ }^{0} / \bar{X}_{n}{ }^{0}=2\right)$ was hypothetically fractionated by means of SPF or SSF and the data $\left(\bar{X}_{w}\right.$ and $\rho$ ) obtained thus were analyzed according to the Schulz or Kamide procedures. Evidently, as $E$ increases the estimated value of $\bar{X}_{w} / \bar{X}_{n}$ tends to scatter to a greater extent and with a gradual decrease in $E$ to zero the value of $\bar{X}_{w} / \bar{X}_{n}$ tends to the limiting value, which is equal to the true value (in this case, $\left.\bar{X}_{w} / \bar{X}_{n}=2\right)$. Both SPF/Schulz and SSF/Schulz have a clear tendency to give smaller values of $\bar{X}_{w} / \bar{X}_{n}$ than the true values (i.e., $\left.\bar{X}_{w}{ }^{0} / \bar{X}_{n}{ }^{0}\right)$. The value of $\bar{X}_{w} / \bar{X}_{n}$ obtained by $\mathrm{SPF} / \mathrm{Kamide}$ is usually larger than the true value, but that estimated by using SSF/Kamide is always lower than $\bar{X}_{w}{ }^{0} / \bar{X}_{n}{ }^{0}$. As $E$ is larger the degree of

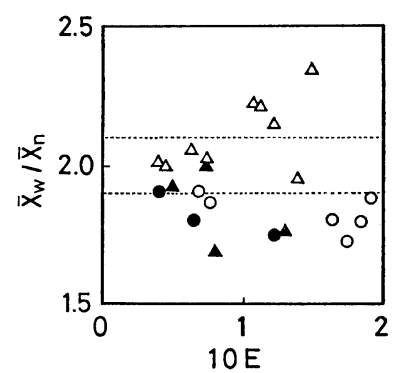

Figure 9. Correlations between the value of $\bar{X}_{w} / \bar{X}_{n}$ of the original polymer, estimated from the fractionation data and the relative error $E$ : The original polymer, Schulz-Zimm distribution SZ 2; open mark, successive precipitational fractionation (SPF); closed mark, successive solutional fractionation (SSF); circle, Schulz method; triangle, Kamide method. 


\section{Y. MiYAZAKI and K. KAMIDE}

deviation of $\bar{X}_{w} / \bar{X}_{n}$ from the true value is larger.

If one wishies to estimate $\bar{X}_{w} / \bar{X}_{n}$ of the original polymer using the fractionation method with an error of $\pm 5 \%$, the conditions should be chosen so that $E$ is, at least, less than $5 \%$. Attention should, however, be drawn to the fact that in the case of SPF fractionation with a precision of $E \simeq 5 \%$ is realizable only under comparatively restricted conditions (see Figure 7). In this connection, SSF/Kamide is recommended for general use.

Experimental Verification of Modern Theory of Analytical Fractionation

MWD curves of the polystyrene fractions isolated by SPF and SSF from a 0.94-\% solution in methylcyclohexane were determined by GPC.

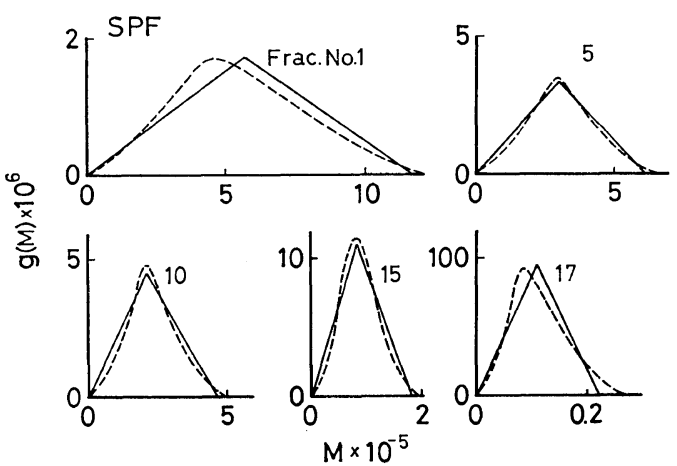

(a)

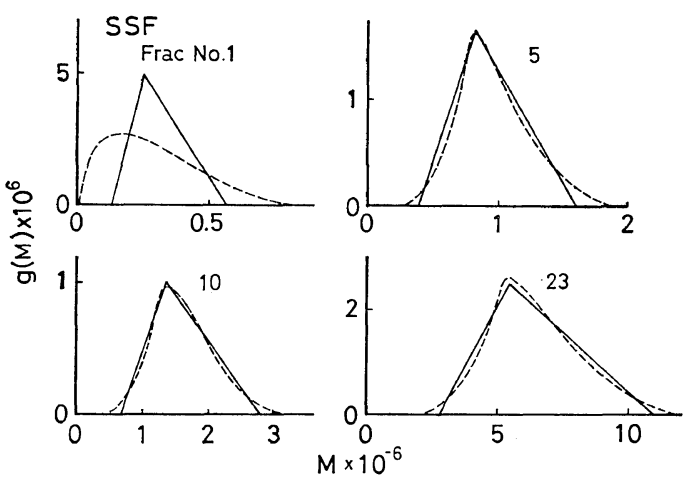

(b)

Figure 10. Comparison of the true molecular weight distribution (MWD) determined by GPC with the triangle-shaped distribution employed in the Kamide's method for atactic polystyrene fractions: (a) successive precipitational fractionation $(\mathrm{SPF}), v_{\mathrm{p}}{ }^{0}=0.94 \%, n_{\mathrm{t}}=18$; (b) successive solutional fractionation (SSF), $v_{\mathrm{p}}{ }^{0}=0.94 \%, n_{\mathrm{t}}=23$.
These were compared with the triangle distribution, which was approximated by Kamide et al., as exemplified in Figure 10. Except for very low-order fraction, the triangle distribution employed by Kamide, et al., proved successful. Therefore, Figure 10 directly supports the validity of the approximations in the Kamide's method.

The maximum and minimum values of the molecular weight, $M_{\max }$ and $M_{\min }$, in the distribution for the polystyrene fractions obtained by SPF and SSF were determined conventionally as the values at which the magnitude of $g_{\mathrm{e}}(M)$ of a fraction is equal to $4 \times 10^{-8}$. In Figure 11 the plot of $M_{\min } / \bar{M}_{w}$ and $M_{\max } / \bar{M}_{w}$ is shown, where $\bar{M}_{w}$ is the weight-average molecular weight. In this figure the results of the computer simulation for this polymer/solvent system are shown. The agreement shown by $M_{\min } / \bar{M}_{w}$ and $M_{\max } / \bar{M}_{w}$ between experiment and theory is fairly satisfactory.

The simplest way of determining $\bar{X}_{w}$ and $\bar{X}_{n}$ is given by:

$$
\bar{X}_{w}=\sum_{j} \rho_{j} \bar{X}_{w, j}
$$

or

$$
\bar{X}_{w}=\sum_{j} \rho_{j} \bar{X}_{n, j}
$$

and

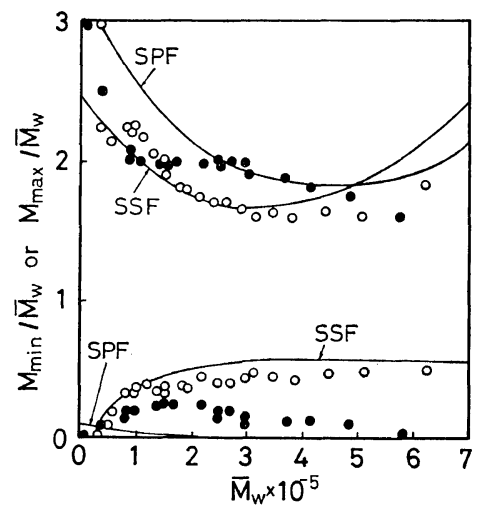

Figure 11. The ratio of $M_{\max } / \bar{M}_{w}$ (and $M_{\min } / \bar{M}_{w}$ ) plotted as a function of $\bar{M}_{w}$ for atactic polystyrene fractions separated by successive solutional fractionation (SSF) $(\bigcirc)$ and successive precipitational fractionation (SPF) (O), experiments; full line denotes the theoretical curves $(p=0.7)$; the original polymer, $\bar{M}_{w}=23.9 \times 10^{4}, \bar{M}_{w} / \bar{M}_{n}=2.77$; solvent, methylcyclohexane; $v_{\mathrm{p}}{ }^{0}=0.94 \%, n_{\mathrm{t}}=23$ in SSF and 18 in SPF. 
Analytical Successive Solutional Fractionation of Macromolecules

Table II. Value of $\bar{M}_{n}, \bar{M}_{w}$, and $E$ evaluated by the actual experiment on polystyrene/methylcyclohexane system (experiment) and the computer simulation with $p=0.7$ (theory)

\begin{tabular}{|c|c|c|c|c|c|c|c|c|c|c|c|}
\hline \multirow{2}{*}{$\begin{array}{l}\text { Fraction- } \\
\text { ation } \\
\text { procedure }\end{array}$} & \multirow{2}{*}{$\begin{array}{c}\text { Operat- } \\
\text { ing } \\
v_{\mathrm{p}^{0}}^{0} \%\end{array}$} & \multirow{2}{*}{$\begin{array}{c}\text { Condi- } \\
\text { tions } \\
n_{\mathrm{t}}\end{array}$} & \multirow{2}{*}{$\begin{array}{l}\text { Analytical } \\
\text { method }\end{array}$} & \multicolumn{2}{|c|}{$\bar{M}_{n} \times 10^{-4}$} & \multicolumn{2}{|c|}{$\bar{M}_{w} \times 10^{-4}$} & \multicolumn{2}{|c|}{$\bar{M}_{w} / \bar{M}_{n}$} & \multirow{2}{*}{$\underset{\text { Expt }}{E^{\prime}}$} & \multirow{2}{*}{$\begin{array}{c}E \\
\text { Theory }\end{array}$} \\
\hline & & & & Expt & Theory & Expt & Theory & Expt & Theory & & \\
\hline \multirow{8}{*}{ SPF } & 0.94 & 13 & eq 9 and 10 & 15.8 & 11.8 & 24.7 & 23.1 & 1.61 & 1.96 & - & - \\
\hline & 0.94 & 13 & eq $9^{\prime}$ and $10^{\prime}$ & 12.1 & 8.8 & 17.4 & 17.4 & 1.44 & 1.98 & - & - \\
\hline & 0.94 & 13 & Schulz & 12.8 & 11.4 & 22.6 & 23.4 & 1.76 & 2.03 & 0.252 & 0.131 \\
\hline & 0.94 & 13 & Kamide & 10.9 & 8.4 & 24.6 & 23.2 & 2.26 & 2.76 & 0.181 & 0.078 \\
\hline & 0.94 & 18 & eq 9 and 10 & 12.5 & 11.3 & 23.1 & 23.1 & 1.85 & 2.04 & - & - \\
\hline & 0.94 & 18 & eq $9^{\prime}$ and $10^{\prime}$ & 10.0 & 8.8 & 18.0 & 17.9 & 1.80 & 2.02 & - & - \\
\hline & 0.94 & 18 & Schulz & 13.1 & 10.8 & 23.8 & 23.7 & 1.81 & 2.20 & 0.222 & 0.156 \\
\hline & 0.94 & 18 & Kamide & 9.5 & 8.7 & 23.1 & 24.1 & 2.43 & 2.77 & 0.123 & 0.103 \\
\hline \multirow{8}{*}{ SSF } & 0.94 & 14 & eq 9 and 10 & 10.4 & 11.5 & 21.7 & 23.1 & 2.10 & 2.00 & - & - \\
\hline & 0.94 & 14 & eq $9^{\prime}$ and $10^{\prime}$ & 8.1 & 8.8 & 19.9 & 21.5 & 2.44 & 2.44 & - & - \\
\hline & 0.94 & 14 & Schulz & 10.6 & 10.6 & 24.8 & 23.1 & 2.34 & 2.16 & 0.091 & 0.068 \\
\hline & 0.94 & 14 & Kamide & 8.9 & 9.5 & 21.9 & 23.0 & 2.46 & 2.41 & 0.095 & 0.068 \\
\hline & 0.94 & 23 & eq 9 and 10 & 11.7 & 10.9 & 22.1 & 23.0 & 1.88 & 2.11 & - & - \\
\hline & 0.94 & 23 & eq $9^{\prime}$ and $10^{\prime}$ & 8.9 & 8.8 & 20.0 & 21.9 & 2.24 & 2.49 & 一 & - \\
\hline & 0.94 & 23 & Schulz & 11.6 & 10.9 & 23.8 & 23.8 & 2.05 & 2.17 & 0.163 & 0.051 \\
\hline & 0.94 & 23 & Kamide & 9.7 & 10.2 & 22.1 & 23.1 & 2.28 & 2.26 & 0.102 & 0.069 \\
\hline GPC & - & - & - & $\begin{array}{c}8.6 \\
(8.9)^{b}\end{array}$ & - & $\begin{array}{c}23.9 \\
(23.2)\end{array}$ & - & 2.77 & - & - & - \\
\hline
\end{tabular}

a $E$ and $E^{\prime}$ was defined by eq 6 and 13 .

b Membrane osmometry.

c Light scattering.

$$
\bar{X}_{n}=1 / \sum_{j} \frac{\rho_{j}}{\bar{X}_{w, j}}
$$

or

$$
\bar{X}_{n}=1 / \sum_{j} \frac{\rho_{j}}{\bar{X}_{n, j}}
$$

where, $\rho_{j}, \bar{X}_{w, j}$ and $\bar{X}_{n, j}$ are the relative amounts, $\bar{X}_{w}$ and $\bar{X}_{n}$ of the $j$ th fraction, respectively. In deriving eq 9 and 10 the polydispersity of the fractions is neglected. In consequence, by use of one kind of average degree of polymerization $\left(\bar{X}_{w, j}\right.$ or $\left.\bar{X}_{n, j}\right)$ one cannot calculate both $\bar{X}_{w}$ and $\bar{X}_{n}$ accurately.

In the strict sense, $\bar{X}_{w}$ and $\bar{X}_{n}$ of the whole polymer should be calculated from;

$$
\begin{aligned}
& \bar{X}_{w}=\int X g_{\mathrm{e}}(X) \mathrm{d} X \\
& \bar{X}_{n}=1 / \int \frac{g_{\mathrm{e}}(X)}{X} \mathrm{~d} X
\end{aligned}
$$

$g_{\mathrm{e}}(X)$ is the differential MWD curve estimated by the Schulz method or the Kamide method.
The values of the number-average molecular weight $\bar{M}_{n}$, of $\bar{M}_{w}$ and of $E$ calculated from the actual and hypothetical fractionation data by SPF and SSF on atactic polystyrene in methylcyclohexane are compiled in Table II. In this table, $E^{\prime}$ of the experiment is conveniently defined by:

$$
E^{\prime}=\int\left|g_{\mathrm{G}}(X)-g_{\mathrm{e}}(X)\right| \mathrm{d} X
$$

Here, $g_{\mathrm{G}}(X)$ is the differential MWD obtained by GPC.

It should be stressed that in the case of computer simulation the relative error $E$ in eq 6 is unreducible error inherent in the fractionation procedure/analytical method and in the experiment a second type of error accompanying the practical determination of $\bar{X}_{w}$ and $\bar{X}_{n}$ should be added to the above-mentioned intrinsic error. Moreover, in the hypothetical fractionations it was assumed that equal amounts of the fractions were separated. Then, a variation of the frac- 


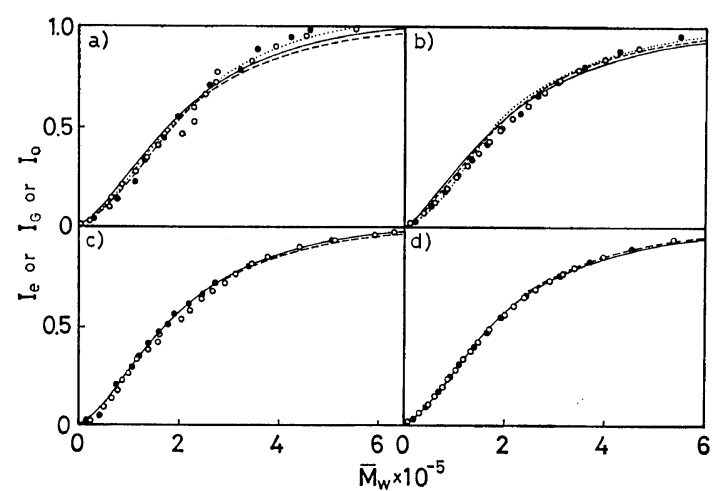

Figure 12. Cumulative weight fraction constructed from the analytical fractionation data, $I_{\mathrm{e}}$ as compared with the true cumulative weight fraction $I_{0}$ and that estimated by GPC, $I_{\mathrm{G}}$ : a) successive precipitational fractionation (SPF), experiment; broken line, $I_{G}$; dotted line, the Kamide method $\left(n_{\mathrm{t}}=13\right)$; full line, the Kamide method $\left(n_{\mathrm{t}}=18\right)$; , the Schulz method $\left(n_{\mathrm{t}}=13\right) ; \bigcirc$, the Schulz method $\left(n_{\mathrm{t}}=18\right)$ : b) successive precipitational fractionation (SPF), theory $(p=0.7)$; broken line, $I_{0}$; other marks have the same meaning as those in Figure 12 a): c) successive solutional fractionation (SSF), experiment; broken line, $I_{G}$; dotted line, the Kamide method $\left(n_{\mathrm{t}}=14\right)$; full line, the Kamide method $\left(n_{\mathrm{t}}=23\right)$; 2 , the Schulz method $\left(n_{\mathrm{t}}=14\right)$; $\bigcirc$, the Schulz method $\left(n_{\mathrm{t}}=23\right)$ : d) successive solutional fractionation (SSF), theory $(p=0.7)$; broken line, $I_{0}$. Other marks have the same meaning as those in Figure $12 \mathrm{c}$ ).

tion size brings about another kind of error to the value of $\bar{X}_{w} / \bar{X}_{n}$. Consequently, the large experimental error makes the detailed comparison with the theory impossible in practice.

The value of $\bar{M}_{n}$ obtained from the fractionation data by means of eq 10, the Schulz method and the Kamide method has, even from the theoretical view point, a large uncertainty compared with the value of $\bar{M}_{w}$ and always appreciably larger than the true value (in this case, $\left.8.6 \times 10^{4}\right)$. The Kamide method is in principle preperable to the Schulz method with respect to the determination of $\bar{M}_{n}$, because the former takes good account of "the tailing effect." The hypothetical fractionation data indicates that the Kamide method without exception affords a more accurate value of $\bar{M}_{w} / \bar{M}_{n}$ than the Schulz method does.

The operating conditions employed here, are appropriate for the preparative fractionation, since the majority of the fraction isolated by SSF has a polydispersity of $\bar{M}_{w} / \bar{M}_{n} \leq 1.10$ (for example, 9(8) and 19(20) fractions have $\bar{M}_{w} / \bar{M}_{n} \leq$ 1.10 at $n_{\mathrm{t}}=14$ and 23 theoretically (experimentally)). As is seen from Figure 12 (a, b, c, d), where $I_{\mathrm{e}}$ and $I_{0}$ or $I_{\mathrm{G}}$ are the cumulative MWD evaluated from the hypothetical fractionation data and that of the original polymer or the cumulative MWD as determined by GPC, the experiment (Figure 12 a and c)) is in satisfactory agreement with the theory (Figure $12 \mathrm{~b}$ and $\mathrm{d}$ )). $\mathrm{SPF} /$ Schulz (Figure 12 a and b)) is shown to be rather less successful even for estimating the MWD curve of the whole polymer, Contrary to this, analytical SSF (Figure $12 \mathrm{c}$ and d)) is very suitable for evaluating the total profile of MWD. The theoretical calculation indicates that the operating conditions used in Table II do not fulfill the requirement of $E<5 \%$. Therefore, in order to estimate accurately the $\bar{M}_{w} / \bar{M}_{n}$ value of the whole polymer, much more serious operating conditions should be chosen carefully.

This prediction was carefully examined by computer simulation. Plots of $\left(\bar{M}_{w} / \bar{M}_{n}\right)_{\mathrm{t}} /\left(\bar{M}_{w} /\right.$ $\left.\bar{M}_{n}\right)_{0} v s . v_{\mathrm{p}}{ }^{0}$, thus obtained, are shown in Figure 13, where $\left(\bar{M}_{w} / \bar{M}_{n}\right)_{\mathrm{t}}$ is the $\bar{M}_{w} / \bar{M}_{n}$ value for the original polymer, evaluated from the SSF data by use of the Schulz or the Kamide method and $\left(\bar{M}_{w} / \bar{M}_{n}\right)_{0}$ is the $\bar{M}_{w} / \bar{M}_{n}$ value for the original polymer. which was assumed in advance in the simulation. Obviously, as $v_{\mathrm{p}}{ }^{0}$ gets small $\left(\bar{M}_{w} / \bar{M}_{n}\right)_{\mathrm{t}} /\left(\bar{M}_{w} / \bar{M}_{n}\right)_{0}$ approaches to unity. The Schulz method have a tendency to give smaller

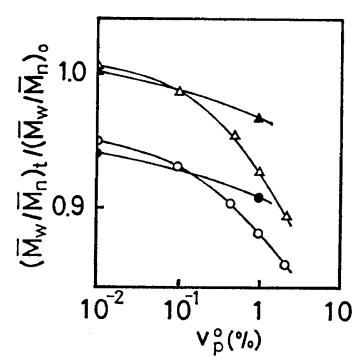

Figure 13. Effect of the initial polymer volume fraction $v_{\mathrm{p}}{ }^{0}$ on the ration $\left(\bar{M}_{w} / \bar{M}_{n}\right)_{\mathrm{t}} /\left(\bar{M}_{w} / \bar{M}_{n}\right)_{0}$ : Circle, the Schulz method; triangle, the Kamide method; open mark, $n_{\mathrm{t}}=20$; closed mark, $n_{\mathrm{t}}=50$; the original polymer, the Schulz-Zimm distribution $\left(\bar{M}_{w}=239000\right.$, SZ 2.77). 
Analytical Successive Solutional Fractionation of Macromolecules

$\left(\bar{M}_{w} / \bar{M}_{n}\right)_{t} /\left(\bar{M}_{w} / \bar{M}_{n}\right)_{0}$ than the Kamide method as was found in Table II. If one wishes to estimate $\bar{M}_{w} / \bar{M}_{n}$ of the original polymer with an error of less than $\pm 5 \%, v_{\mathrm{p}}{ }^{0} \leq 0.5 \%$ at $n_{\mathrm{t}}=20$ or $v_{\mathrm{p}}{ }^{0} \leq$ $2 \%$ at $n_{\mathrm{t}}=\mathbf{5 0}$ as the suitable operating conditions should be chosen in the case of the Kamide method. In contrast, if the Schulz method is employed, the operating conditions of $v_{\mathrm{p}}^{0} \geq 0.01 \%$ and $n_{\mathrm{t}} \leq 50$ do not fulfill the above requirement of $0.95<\left(\bar{M}_{w} / \bar{M}_{n}\right)_{t} /\left(\bar{M}_{w} / \bar{M}_{n}\right)_{0}<1.05$.

Unfortunately, the value of $\bar{X}_{w} / \bar{X}_{n}$ (or $\bar{M}_{w} / \bar{M}_{n}$ ) obtained from the analytical fractionation data by means of eq 9 and 10 or by the Schulz method has so far been compared directly only with the theoretical value, which has been derived by polymerization theory, and has been correlated with some mechanical properties. It is obvious from Table II that much attention should be paid, especially when $\bar{X}_{w} / \bar{X}_{n}$ of the original polymer is to be determined from the fractionation data. In conclusion, a combination of SSF and the Kamide method is strongly recommended for estimating the MWD of the original polymer.

\section{REFERENCES}

1. See, for example, J. N. Cardenas and K. F. O' Driscoll, J. Polym. Sci., Polym. Lett., 13, 657 (1975).

2. D. Berek, D. Bakos, T. Bleha, and L. Soltes, Makromol. Chem., 176, 391 (1975).

3. J. V. Dawkins and M. Hemming, ibid., 176, 1777 (1975).

4. J. V. Dawkins and M. Hemming, ibid., 176, 1795 (1975).

5. J. V. Dawkins and M. Hemming, ibid., 176, 1815 (1975).
6. M. R. Ambler and D. McIntyre, J. Polym. Sci., Polym. Lett., 13, 589 (1975).

7. See, for example, K. Kamide, High Polymer Summer Symposia, 1972, p 117.

8. M. Matsumoto and Y. Ohoyanagi, Kobunshi Kagaku (Chem. High Polymers), 11, 7 (1954).

9. C. Booth and L. R. Beason, J. Polym. Sci., 42, 81 (1960).

10. L. H. Tung, ibid., 61, 449 (1962).

11. See, for example, K. Kamide, Kobunshi Ronbunshu, 31, 147 (1974).

12. K. Kamide and Y. Miyazaki, Makromol. Chem., 176, 1029 (1975).

13. K. Kamide and Y. Miyazaki, ibid., 176, 1051 (1975).

14. K. Kamide and Y. Miyazaki, ibid., 176, 1427 (1975).

15. K. Kamide and Y. Miyazaki, ibid., 176, 1447 (1975).

16. K. Kamide and Y. Miyazaki, ibid., 176, 2393 (1975).

17. K. Kamide and Y. Miyazaki, ibid., 176, 3453 (1975).

18. K. Kamide, K. Sugamiya, T. Ogawa, C. Nakayama and N. Baba, ibid., 135, 23 (1970).

19. K. Kamide, K. Sugamiya, T. Terakawa, and T. Hara, ibid., 156, 287 (1972).

20. K. Kamide, Y. Miyazaki, and T. Abe, ibid., 177, 485 (1976).

21. K. Kamide and K. Sugamiya, ibid., 156, 259 (1972).

22. K. Kamide and K. Sugamiya, ibid., 139, 197 (1970).

23. G. V. Schulz and A. Dinglinger, Z. Phys. Chem., B43, 47 (1939).

24. K. Kamide, T. Ogawa, M. Sanada, and M. Matsumoto, Kobunshi Kagaku (Chem. High Polymers), 25, 440 (1968).

25. K. Kamide, Y. Miyazaki, and K. Yamaguchi, Makromol. Chem., 173, 175 (1973). 